Document downloaded from:

http://hdl.handle.net/10251/94474

This paper must be cited as:

Juan Borras, MDS.; Soto Camino, J.; Gil Sánchez, L.; Pascual-Maté, A.; Escriche Roberto, MI. (2017). Antioxidant activity and physicochemical parameters for the differentiation of honey using a potentiometric electronic tongue. Journal of the Science of Food and Agriculture. 97(7):2215-2222. doi:10.1002/jsfa.8031

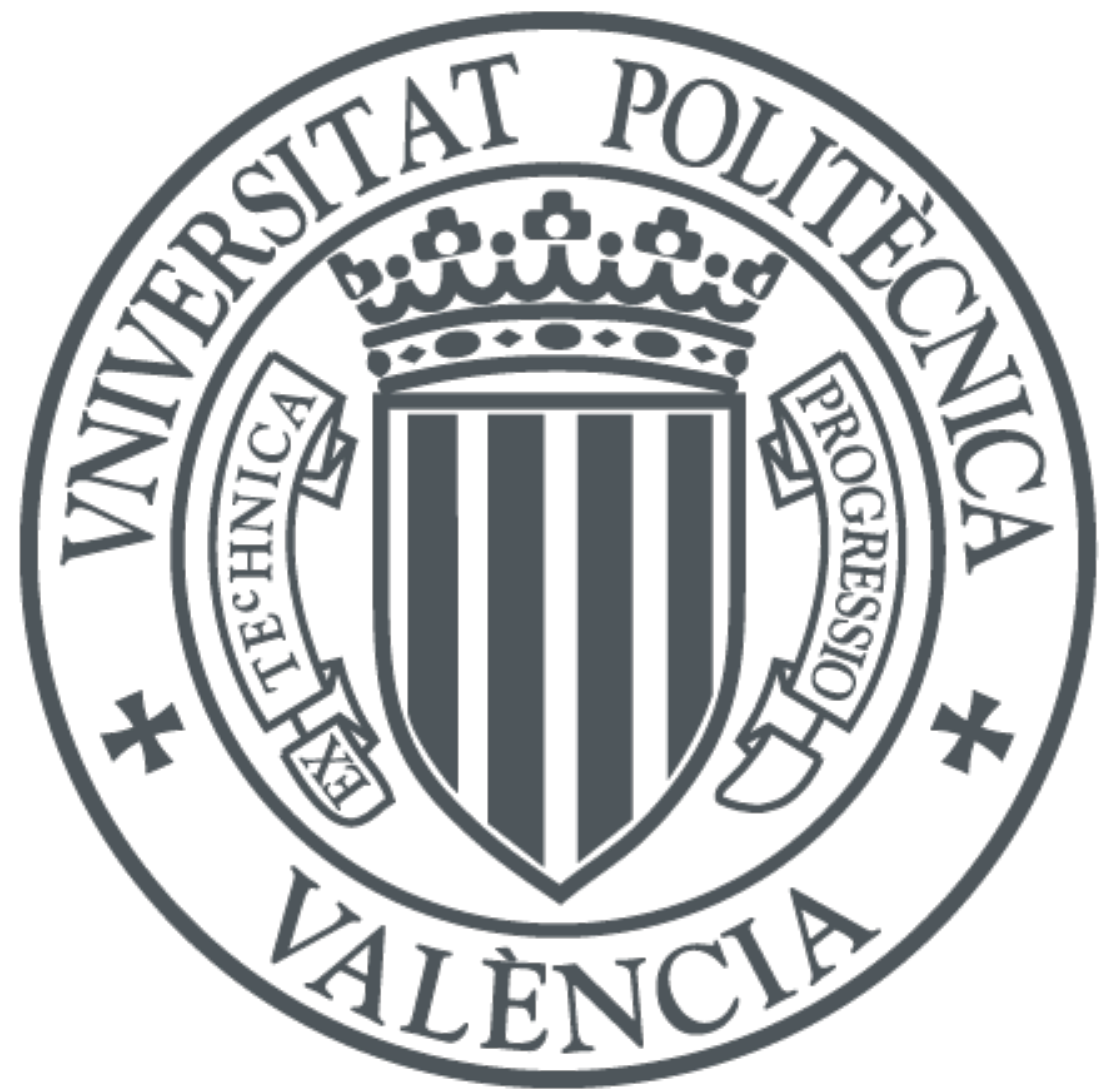

The final publication is available at

http://doi.org/10.1002/jsfa.8031

Copyright John Wiley \& Sons

Additional Information 


\title{
Antioxidant activity and physicochemical parameters for the differentiation of honey using a potentiometric electronic tongue
}

Marisol Juan-Borrás ${ }^{a}$, Juan Soto b,c , Luis Gil-Sánchez ${ }^{\text {b,d }}$, Ana Pascual-Maté e, Isabel Escriche $^{\mathrm{a} *}$

${ }^{a}$ Institute of Food Engineering for Development (IUIAD). Food Technology Department (DTA). Universitat Politècnica de València. P.O. Box 46022 Valencia, Spain.

${ }^{b}$ Instituto de Reconocimiento Molecular y Desarrollo Tecnológico (IDM), Centro Mixto Universitat Politècnica de València - Universidad de Valencia, Camino de Vera s/n, 46022 Valencia, Spain.

${ }^{c}$ Departamento de Química, Universidad Politécnica de Valencia, Camino de Vera s/n, E-46022 Valencia, Spain

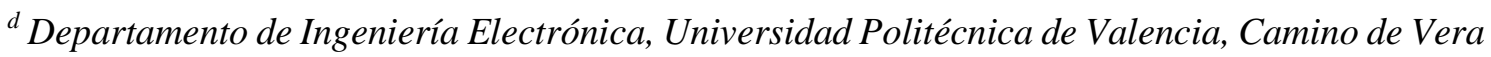
s/n, E-46022 Valencia, Spain

${ }^{f}$ Department of Biotechnology and Food Science, University of Burgos, Plaza Misael Bañuelos s/n, 09001 Burgos, Castilla-León, Spain.

*Corresponding author: Isabel Escriche, iescrich@tal.upv.es;

Tel.: +34-963877366; fax: +34-963877369

\begin{abstract}
BACKGROUND: This work evaluates the capacity of a potentiometric electronic tongue to differentiate between types of honey (orange blossom, rosemary, thyme, sunflower, winter savory and honeydew honey) according to their antioxidant level. The classical procedures used to evaluate the antioxidant potential of honey, are inappropriate for in situ monitoring on reception of batches of raw honey in the packaging industry, are destructive, time-consuming and very tedious, requiring highly expert analysts and specialized equipment.
\end{abstract}


RESULTS: The electronic tongue system made with Ag, Ni, Co, $\mathrm{Cu}$ and $\mathrm{Au}$ was able to not only differentiate between types of honey but also to predict their total antioxidant capacity. The discrimination ability of the system was proved by means of a fuzzy ARTMAP type ANN, with $100 \%$ classification success. A prediction MLR model showed that the best correlation coefficient was for antioxidant activity (0.9666), then for electrical conductivity (0.8959) and to a lesser extent for $\mathrm{a}_{\mathrm{w}}$, moisture and colour.

CONCLUSION: The proposed measurement system could be a quick, easy option for the honey packaging sector to provide continuous in line information about a characteristic as important as the antioxidant level.

Keywords: Potentiometric tongue; honey; antioxidant capacity

\section{INTRODUCTION}

Honey has been a highly valued food since ancient times for its organoleptic and therapeutic characteristics and also nowadays for its antioxidant properties. The demand for natural antioxidants is increasing constantly as they play an important role in human health avoiding damage caused by oxidizing agents. It is well known that honey is an important source of natural antioxidants, one of the reasons why honey consumption is recommended. Recent research has shown that the antioxidant properties of honey depend on the nectar of blossoms or exudates of trees and plants visited by bees. In turn, this is conditioned by the geographical and climatic conditions. ${ }^{1}$ At present, the information available to the consumer through labeling is very incomplete. This is limited at the most to the botanical origin, allowing honey to be classified as monofloral. There is growing interest in assessing the specific healthy properties of honey (e.g. antioxidant level), in order to inform consumers and to increase producers profit margins. 
A large number of procedures are used to evaluate the antioxidant potential of honey. Although these methodologies show good precision, accuracy and reliability, the problem is that they are inappropriate for in situ monitoring on reception of batches of raw honey in the packaging industry, as they require trained personnel and time to carry out the analysis. In addition, these techniques are destructive, time-consuming and very tedious, requiring highly expert analysts and specialized equipment. ${ }^{2}$ Among the fastest, easy-tohandle measurement techniques that have been tested in recent years to differentiate types of honey are electronic tongues based on: potentiometry, (commercially available as Astree, Alpha M.O.S or homemade) ${ }^{3,4,5,6 ;}$; voltammetry ${ }^{7}$; impedance spectroscopy ${ }^{8}$ or a combination of these techniques ${ }^{9}$. Whatever the technique applied, the great amount of data produced must be always treated using appropriate multivariate analysis techniques such as: PCA (principal component analysis) ${ }^{10}$; LDA (linear discriminant analysis) ${ }^{11}$, CA (cluster analysis); ANN (artificial neural network) ${ }^{12}$ or (MLR) Multiple linear regression ${ }^{13}$.

Using potentiometric electrodes provides information about the presence of compounds through interaction with them. ${ }^{14}$ For example, metal/metal oxide electrodes are sensors that afford information about the $\mathrm{pH}$ of the medium, the presence of anions and the presence of dissolved substances which can form compounds with the corresponding metals. ${ }^{4}$ The measurement of the redox potential (using noble metals) can be directly related to the content of total reducing or oxidizing agents present in the sample. Antioxidants, such as polyphenols or flavonoids, are compounds with reducing character that can cause changes in the potential of metal polarization as a consequence of both their potential redox and their relative concentrations. ${ }^{14}$ The connection between the antioxidant activity and the electroanalysis techniques has been succefully proven in different kinds of food. ${ }^{15}$ 
This suggests that metal sensors could be useful for the determination of the antioxidant activity of honey.

For this reason the goal of the present study was to evaluate whether a potentiometric electronic tongue made with a combination of metal electrodes (noble and non-noble) is able to differentiate between types of Spanish honey according to their antioxidant level. The specific contribution of each electrode sensor was evaluated, together with the possibility of a correlation between the potentiometric measures of honey and the physicochemical parameters and antioxidant capacity.

\section{MATERIAL AND METHODS}

\section{Honey samples}

Six types raw honey harvested in 2014 in different areas of Spain were used in this study. They are among the most common varieties available in Spain: orange blossom (Citrus spp.), rosemary (Rosmarinus officinalis), thyme (Thymus spp.), sunflower (Helianthus annuus), winter savory honey (Satureja montana), and honeydew honey. Three batches of each type of honey were directly supplied by beekeepers. For each type of honey, 3 different beekeepers provided 1 batch each. Each beekeeper supplied $25 \mathrm{~kg}$ of honey taken from their stock of at least $10000 \mathrm{~kg}$ of honey for each variety.

\section{Melissopalynological analysis.}

The botanical categorization of each of the batches were performed by means of pollen analysis, which was quantified following the recommendations of the International Commission for Bee Botany. ${ }^{16}$ The interlaboratory study carried out in this research showed that the coefficient of variation $\mathrm{RSD}_{\mathrm{R}} \%$ of this method is $29.8,32.4$ or 19.3 , depending on the presence of absolute pollen number: low, medium and high, respectively. Microscopic examination (at 400 to 1000x magnification), identification 
and the interpretation of pollen types were carried out by an experienced pollen analyst, using pollen slides and references ${ }^{17,18}$.

\section{Colour and physicochemical analysis}

Color was determined using a millimeter Pfund scale C 221 Honey Color Analyzer (Hanna Instruments). Moisture content (by refractrometry: Abbe-type model T1 Atago, USA and the Chataway table) and electrical conductivity (by conductimetry: Crison Instrument, Barcelona, Spain, model C830) were analyzed in accordance with the Harmonized Methods of the European Honey Commission (Bogdanov, 2002). The precision of these analytical methods (expressed as the coefficient of variation $\mathrm{RSD}_{\mathrm{R}} \%$ ) was reported by the International Honey Commission in 2009, taking into account the results of an interlaboratory study. ${ }^{19}$

Water activity $\left(\mathrm{a}_{\mathrm{w}}\right)$ was determined at $25^{\circ} \mathrm{C}\left( \pm 0.2^{\circ} \mathrm{C}\right)$ using an electronic dewpoint water activity meter, Aqualab Series 4 model TE (Decagon Devices, Pullman,Washington, USA), equipped with a temperature-control system. ${ }^{20}$

\section{Sugar Analysis}

Fructose, glucose and sucrose were analyzed as described by Bogdanov, et al. (1997) using a HPAEC high-resolution ionic chromatograph with a pulsed amperometric detector (PAD) (Bioscan, Methrom, Switzerland) and a Metrosep Carb chromatographic column (styrene divinylbenzene copolymer, $4.6 \times 250 \mathrm{~mm}$ ). ${ }^{21}$ Quantification of sugars was realized using external standards constructing the corresponding calibration curves. The quantification limits of the tree sugars studied were $0.1 \mathrm{~g} / 100 \mathrm{~g}$ honey.

\section{Antioxidant activity}

The antioxidant activity (AA) was measured based on the scavenging activities of the stable DPPH (2,2-diphenyl-1-picrylhydrazyl) (Sigma-Aldrich, Germany) free radical. ${ }^{22}$ 
$0.3 \mathrm{~mL}$ honey solution $(0.1 \mathrm{~g} / \mathrm{mL})$ was mixed with $2.7 \mathrm{ml} \mathrm{DPPH}$ solution $\left(6 \times 10^{-5} \mathrm{~mol} / \mathrm{L}\right)$; after 30 minutes in the dark at room temperature, the absorbance of the solutions was measured at $517 \mathrm{~nm}$ ). Results were expressed as mg of trolox equivalent per 100 gram of honey.

All analyses (colour, physicochemical, sugar and antioxidant activity) were performed in triplicate.

\section{Potentiometric tongue}

Measurement system. Measurements were performed with tailor-made equipment formed basically by the following parts: a set of metal electrodes, an electronic system ${ }^{23}$, a computer system for digitalization and acquisition of the signal and data analysis software.

Set of metallic electrodes. The electrochemical measurements were obtained using an electronic tongue consisting of an array of eight metallic electrodes (1 mm diameter from Aldrich): Noble metals (gold, platinum, iridium and rhodium); and non-noble metals (copper, silver, nickel and cobalt). All electrodes were placed in two homemade stainless steel tube (one for the 4 noble metals and another for the rest) that were used as the body of the electronic tongue. The different wire electrodes were fixed inside the tube using RS 199-1468 epoxy polymer. Before use, the surfaces of the electrodes were polished with emery paper, and rinsed with distilled water. Then they were polished on a felt pad with $0.05 \mu \mathrm{m}$ alumina polish from BAS, washed with distilled water and polished again on a nylon pad with 15.3 and $\mu \mathrm{m}$ diamond polishes. During the measurements, only a simple diamond polishing was carried out and washed with distilled water.

Electronic Measurement Equipment. The measuring equipment permitted multiplexed potentiometric measures for eight independent channels. The potential was measured 
automatically, sequentially and cyclically for each of the eight electrodes with respect to the $\mathrm{Ag} / \mathrm{AgCl}$ reference electrode. The potentiometric measurements were simply the potential difference between each electrode and the reference electrode. The set formed by the electrodes and the sample container was introduced in a thermostatic bath system at $25.0^{\circ} \mathrm{C}$. The electronic system has two stages, firstly, an analogue circuit and then a digital system. The analogue stage is formed by very high impedance electrometric amplifiers LMC6001 and active low-pass filters to remove the noise from the electrical network. The measurement assembly was incorporated into a Faraday cage that was connected to the ground of the building. The second stage is composed of an Adlink PCI9112 card for data acquisition and further analysis. Analogic-digital converters and VEEPro software (version 9.0) were used for viewing the data on the computer screen and storing the data for subsequent processing. The card is a 12 bits $\mathrm{A} / \mathrm{D}$ converter with a reference voltage of $5 \mathrm{~V}$ reaching a resolution of $1.22 \mathrm{mV}$.

Implementation of measures. To carry out a measurement, the set of eight electrodes and the reference electrode were dipped in a vessel containing $5 \mathrm{~g}$ of honey in $25 \mathrm{~mL}$ water. Then, the multiplexed reading of the potential was carried out for $15 \mathrm{~min}$. The time required to reach equilibrium was usually less than 10 minutes. The data used for multivariate analysis were the average values of the last 5 min of acquisition (between 10 and $15 \mathrm{~min}$ ). This process was repeated three times for each of the 18 samples (6 types of honey x 3 batches). Samples were always measured in random order.

Statistical Analysis. An analysis of variance (ANOVA) (using Statgraphics Centurion 16.1) was applied to study the influence of the type of honey on colour, physicochemical parameters $\left(\mathrm{a}_{\mathrm{w}}\right.$, moisture and electrical conductivity), sugars and total antioxidant activity. LSD (least significant difference) at significance level $\alpha=5 \%$ was used to analyze the differences between means. 
The data matrix obtained with the potentiometric tongue was analyzed using multivariate statistical techniques: principal component analysis (PCA) and Fuzzy ARTMAP-type artificial neural network (ANN) were applied to evaluate the possible classification (nonsupervised and supervised, respectively) of the samples. Fuzzy ARTMAP type ANN (Carpenter, et al., 1992) is a self-organizing supervised classifier used with electronic nose and tongue systems. ${ }^{24}$ This classifier produces good results with a limited number of samples. ${ }^{25,26}$

The operation of this neural network requires control parameters, which must be adjusted in the training stage. The main parameters are: surveillance, which indicates the similarity of the elements in the same category; and learning speed, which indicates the weighting of the new data entering the network.

The network was implemented in-house using function macros from basic functions of MATLAB (version R2010a) with a graphical environment (GUI) ${ }^{27}$ using a simplified version of the original algorithm ${ }^{28}$ (Kasuba, 1993). Multiple linear regression (MLR) was used to model the relationship between the potentiometric measurement (explanatory variables) and the physicochemical parameters (response variables). PCA and MLR was performed using the annex SOLO of the MATLAB program (Eigenvectors Research, Inc.) (version 7.0.3). An auto scaling of the input data was realized in order to give equal weight to all electrodes in the model. Cross-valiation (Venetian-Blinds type) was applied to create the model.

\section{RESULTS AND DISCUSSION}

Melissopalynological, colour, physicochemical parameters, sugars and antioxidant activity characterization

Pollen analysis of the honey samples was made as a first step in order to ascertain their botanical origin. Table S1 illustrates the dominant and accompanying pollen of each 
sample, colour, physicochemical parameters, sugars and total antioxidant activity of each honey sample used in this study. The percentage of dominant pollen has been included in brackets as it defines the botanical type of honey. In addition, this table shows the ANOVA results (F-ratio and significant differences) obtained for the factor "type of honey”. The information about the homogeneous groups obtained from the ANOVA for every one of the parameters appears (in superscript letters) in this table next to the information of the first batch of each type of honey. Taking into account that the higher the F-ratio, the greater the effect that a factor has on a variable, electrical conductivity, colour and total antioxidant activity were the parameters most affected by "type of honey”. Moisture, $a_{w}$, and glucose where less affected, whereas fructose and sucrose did not show significant differences between types of honey.

Among all the honey samples considered in this study, honeydew honey, as expected, had the highest electrical conductivity (up to $814 \mu \mathrm{S} / \mathrm{cm}$ ). In fact, this honey has to have more than $800 \mu \mathrm{S} / \mathrm{cm}$ to be classed as this type of honey. Thyme honey standed out from the other monofloral honeys due to its high electrical conductivity values, which reached 517 $\mu \mathrm{S} / \mathrm{cm}$. This is a high value considering its floral origin. This was observed by other authors in thyme honey from Greece (average value: $399 \mu \mathrm{S} / \mathrm{cm}$ ) (Karabagias, et al. (2014). Orange blossom and rosemary honey had the lowest conductivity (no more than 150 and $178 \mu \mathrm{S} / \mathrm{cm}$, respectively), without significant differences between them.

With respect to colour, the results show that orange blossom and rosemary honey had the lightest colour with a range of 0 to $16 \mathrm{~mm}$ Pfund, and winter savory honey the darkest showing values up to $90 \mathrm{~mm}$ Pfund. The dark colour in honey is traditionally a highly valued characteristic because it is well known that the darker a honey is, the higher the mineral content, which is reflected in higher conductivity (González-Miret, et al., 2005; 12. Furthermore, this tendency is usually extrapolated to antioxidant activity, to the point 
that in general the darker the color and conductivity, the higher the antioxidant capacity (Sousa, et al., 2014). However the results of the present work do not support this idea. In fact, although the lowest values of total antioxidant activity correspond to the lightest honeys (orange blossom and rosemary honeys), the darkest honey (winter savory honey) did not exhibit the greatest antioxidant activity. Of all the analyzed samples, thyme honey differs significantly in relation to antioxidant activity, as the values of this parameter were between 4-5 times more than that of winter savory honey and 5-7 times more than honeydew honey, traditionally considered to have a high antioxidant level. The highest values of antioxidant activity in thyme honey could be attributed to the high vitamin $\mathrm{C}$ content present in this type of honey (León-Ruiz, et al., 2011).

The PCA bi-plot of scores and loading obtained considering the 18 different samples (6 types of honey x 3 batches), the physicochemical parameters and color variables is shown in Figure 1, with the aim of evaluating the global effect of the type of honey on these variables from a descriptive position. The percentage of predominant pollen is included next to the code for each sample.

Two principal components explained $66 \%$ of the variations in the data set: PC1 (44\%) and PC2 (22\%). It can be observed that honeydew honey and thyme honey are located in the right quadrant (although not totally differentiated), next to the highest values of colour, electrical conductivity and antioxidant activity. The other samples on the left quadrant without a clear distinction between them. Although the botanical origin of honey had a clear impact on some of the parameters studied, in general terms a good differentiation between the types of honey studied was not achieved.

Potentiometric electronic tongue information: PCA and artificial neural networks classifications 
A PCA analysis (unsupervised procedure) was applied to check if there was a spontaneous classification from the data generated by the eight electrodes, without previously defining the categories of the samples. Figure S1 shows the PCA biplot (scores and loadings) from the measurements obtained with the eight electrodes together, noble and non-noble metals. Two components explained $92 \%$ of the total variance (PC1=72\% and PC2=20\%). It can be observed that a good discrimination was found between types of honey. Samples of thyme honey and honeydew honey showed the clearest separation between them and with the rest of the samples; PC1 promotes the best discrimination of thyme honey and PC2 of the honeydew honey. This figure also shows that sunflower and winter savory are well differentiated, whereas citrus and rosemary have the most similar behavior in terms of potentiometric tongue response.

With respect to the contribution of each electrode in the calculation of PC1 and PC2 values, it can be seen that two electrodes ( $\mathrm{Co}$ and $\mathrm{Cu}$ ) have very different behavior to the rest. This is particularly important with regards to their influence on the PC2, since the variation of the contribution of these two electrodes is not relevant to PC1. This figure shows that the location of the four noble metal electrodes ( $\mathrm{Au}, \mathrm{Pt}$, Ir and $\mathrm{Rh}$ ) are very close to each other, which implies that their behavior is quite similar. Furthermore, the position of these four metals is relatively close to the other two non-noble metals: especially Ag, and to a lesser extent Ni. Consequently, it was considered appropriate to simplify the electrode system, reducing the four noble metals to one, Au, which was selected because is easier to obtain and to work with in comparison to the other three noble metals (Pt, Ir, Rh). Therefore, a new PCA was carried out (Figure 2) only using the information from five electrodes: the four non-noble metals (Ag, Ni, Co, Cu) plus $\mathrm{Au}$. It can be observed that the position of the samples in Figure 2 is quite similar to Figure S1. In the case of Figure 2 the three noble electrodes: $\mathrm{Au}, \mathrm{Ag}$ and $\mathrm{Ni}$, exhibited similar but 
slightly different behavior, while the other two metals ( $\mathrm{Co}$ and $\mathrm{Cu}$ ) still presented different behavior to each other and the rest. In this way it was possible to simplify the measure system without losing information.

With the aim of complementing the information given by the PCA, an artificial neural network analysis (ANN) was applied as a supervised procedure. The ANN is a good tool to predict the botanical origin of honeys using physical and chemical parameters. ${ }^{12}$ The type of neural network used in this work was Fuzzy Artmap (Carpenter, et al., 1992). In this analysis the available data were divided into two groups; one group was used for network training and obtaining the prediction model of the categories, and the other group to verify the neural network and to estimate the degree of success in the classification of new data into the established categories. The data distribution was as follows: of the 54 total input data (6 types of honey x 3 batches x 3 repetitions), two-thirds (36) of the data were used for training and a third (18) for validation; ensuring that each category (types of honey) was represented proportionally in both the training and the validation groups. That is to say, for training the matrix of measurements of each electrode was: 6 types of honey x 2 batches $\mathrm{x} 3$ repetitions, and for validation: 6 types of honey $\mathrm{x} 1$ batches $\mathrm{x} 3$ repetitions.

The optimum model was achieved by obtaining a scan of the operating parameters of the neural network. After that, the data of verification were applied to check the rate of success/failures for the identification of each sample in the appropriate category. The classification success (data not shown) was 100\%. The same result was obtained considering the starting 8 electrodes ( 4 noble and 4 non-noble metals). This demonstrates that the electrode measurement system made with the 5 metals in combination to the ANN is able to correctly classify samples of honey depending on the floral origin, since 
artificial neural networks activate the output of the corresponding category (type of honey).

3.3. Correlation of potentiometric data with physicochemical parameters: MLR analysis. With the aim of verifying whether the data given by the electronic tongue system is useful to predict relevant information provided by the physicochemical parameters, a MLR analysis (Multiple Linear Regression) was applied.

To perform the MLR analysis, the data of 36 samples were taken to create the prediction model and the remaining 18 samples were used to verify the performed model. That is to say, the same separation of samples previously used in the neural network analysis.

A prediction MLR model was applied to the potentiometric experimental data from the metallic electrodes for each the physicochemical parameters ( $a_{w}$, conductivity, moisture), colour and antioxidant activity. The analysis was made twice, on one hand considering the eight electrodes (4 noble metals plus 4 non-noble metals) and on the other hand considering just the 5 selected electrodes (4 non-noble metals plus $\mathrm{Au}$ ). Table 1 shows the MLR results including the values of the correlation coefficient, slope, and intercept obtained from the graphical plot of real measures against those predicted by the model. A good correlation exists for total antioxidant activity and electrical conductivity. The best correlation coefficient was for the antioxidant activity using the 4 non-noble metals plus $\mathrm{Au}$ (0.9666), although it was very similar to that obtained with all the electrodes (0.9665). In the case of electrical conductivity, the correlation coefficients were 0.8959 and 0.9049, respectively. A weaker correlation exists for the rest of the parameters, especially for $\mathrm{a}_{\mathrm{w}}$.

A previous study found a remarkable correlation between physicochemical parameters such as colour Pfund (0.9580) and diastase activity (0.9260) and data from an electronic tongue, made of three metals $(\mathrm{Au}, \mathrm{Ag}$ and $\mathrm{Cu})$ and four metal compounds $\left(\mathrm{Ag}_{2} \mathrm{O}, \mathrm{AgCl}\right.$, 
$\mathrm{Ag}_{2} \mathrm{CO}_{3}$ and $\left.\mathrm{Cu}_{2} \mathrm{O}\right) .{ }^{4}$ However, the correlation with total antioxidant activity was much weaker (0.7660).

Electronic tongues have been used to differentiate different types of teas according to antioxidant activity (Buratii 2013)or a characteristic such as color (Sousa, et al., 2014). However, as far the authors know, this is the first time that a potentiometric electronic tongue has been applied to differentiate between types of honey according to their antioxidant level. The new electronic tongue proposed here is a great improvement over the previous one from the point of view of its ability to differentiate honey considering the antioxidant capacity.

J Agric Food Chem. 2007 Oct 31;55(22):8958-66. Epub 2007 Oct 2.

Evaluation of antioxidant activity and electronic taste and aroma properties of antho-beers from purple wheat grain

\section{CONCLUSION}

An electronic tongue system made of 5 metals: 4 non-noble metals (copper, silver, nickel and cobalt) and just one noble metal (Au) is able to not only differentiate between types of honey but also to predict their total antioxidant capacity.

The differentiation was most marked for thyme and honeydew honey and much weaker for citrus and rosemary honey. The discrimination ability of the measurement system was evaluated by means of an ANN fuzzy artmap type analysis, showing that the classification success was $100 \%$. 
The significance of this study could be the application of the proposed measurement system for the honey packaging industry to provide fast continuous information about a factor as relevant as antioxidant capacity. The importance of knowledge about this component in honey is increasing, not only due to its implications for health, but also in terms of marketing.

This automatic tongue system has produced promising results which could lead to its use in online systems. However, further research is required in order to tackle problems such as the implementation of an automatic cleaning system.

\section{ACKNOWLEDGMENT}

The authors thank the Generalitat Valenciana (Spain) and the Spanish Government for funding the projects AICO/2015/104 and (MAT2015-64139-C4-3-R), respectively.

\section{REFERENCES}

1. Escriche, I., Kadar, M., Juan-Borrás, M., \& Domenech, E. (2014). Suitability of antioxidant capacity, flavonoids and phenolic acids for floral authentication of Spanish honey. Impact of industrial thermal treatment. Food Chemistry, 142, 135143.

2. Oroian, M. \& Escriche, I. (2015). Antioxidants: characterization, natural sources, extraction and analysis. Food Research International, 74, 10-36.

3. Major, N., Markovic, K., Krpan, M., Saric, G., Hruskar, M. \& Vahcic, N. (2011). Rapid honey characterization and botanical classification by an electronic tongue. Talanta, 85, 569-574.

4. Escriche, I., Kadar, M., Domenech, E., \& Gil-Sánchez, L. (2012). A potentiometric electronic tongue for the discrimination of honey according to the botanical origin. 
Comparison with traditional methodologies: Physicochemical parameters and volatile profile. Journal of Food Engineering, 109, 449-456.

5. Qiu, S., Wang, J. \& Gao, L. (2015), Qualification and quantization of processed strawberry juice based on electronic nose and tongue, LWT - Food Science and Technology, 60, 115-123.

6. Newman, J., O'Riordan, D., Jacquier, J.C. \& O'Sullivan, M. (2015). Masking of bitterness in dairy protein hydrolysates: Comparison of an electronic tongue and a trained sensory panel as means of directing the masking strategy, LWT - Food Science and Technology, 63, 751-757.

7. Tiwari, K., Tudu, B., Bandyopadhyay, R., \& Chatterjee, A. (2013). Identificattion of monofloral honey using voltammetric electronic tongue. Journal of Food Engineering, 117, 205-210.

8. Ulloa, P. A., Guerra, R., Cavaco, A.M., Rosa da Costa, A. M. \& Figueira, A.C. (2013). Determination of the botanical origin of honey by sensor fusion of impedance e-tonjgue and optical spectroscopy. Computers and Electronics in Agriculture, 94, 1-11.

9. Wei, Z. \& Wang, J. (2014). Tracing floral and geographical origins of honeys by potentiometric and voltammetric electronic tongue. Computers and Electronics in Agriculture, 108, 112-122.

10. Buratti,S., Sinelli, N., Bertone, Venturello, A., Casiraghia, E. and Geobaldo, F. Discrimination between washed Arabica, natural Arabica and Robusta coffees by using near infrared spectroscopy, electronic nose and electronic tongue analysis. (2014).

11. Sousa, M.E.B.C., Dias, L. G., Veloso, A. C.A., Estevinho, L., Peres, A. M. \& Machado, A.A.S.C. (2014). Practical procedure for discriminating monofloral 
honey with a broad pollen profile variability using an electronic tongue. Talanta, 128, $284-292$.

12. Anjos, O., Peres, Iglesias, C., Martínez, F. J., García, A., \& Taboada, J. (2015). Neural networks applied to discriminate botanical origin of honeys. Food Chemistry, 175, 15, 128-136.

13. Dias, L. G., Veloso, A.C.A., Sousa, M.E.B.C., Estevinho, L., Machado, A.A.S.C. \& Peres, A. M. (2015). A novel approach for honey pollen profile assessment using an electronic tongue and chemometric tools. Analytica Chimica Acta, 900, 5, 36-45.

14. Soto, J., Labrador, R. H., Marcos, M. D., Martınez-Máñez, R., Coll, C., GarcíaBreijo, E. \& Gil, L. (2006). Introduction of a model for describing the redox potential in faradic electrodes. Journal of Electroanalytical Chemistry, 594, 96104.

15. Eric S. Gil,1 Renê O. Couto. Flavonoid electrochemistry: a review on the electroanalytical applications,2 Revista Brasileira de Farmacognosia Brazilian Journal of Pharmacognosy 23(3): 542-558, May/Jun. 2013

16. Von Der Ohe, W., Persano Oddo, L., Piana, M. L. Morlot, M. \& Martin, P. (2004). Harmonized methods of melissopalynology, Apidologie, 35, 18-25.

17. Sáenz C. \& Gómez C. (2000). Mieles españolas. Características e identificación mediante el análisis del polen. Ediciones Mundi-Prensa. Madrid (España).

18. Persano-Oddo L. \& Piro R. (2004). Main European unifloral honeys: descriptive sheets, Apidologie 35 (Suppl. 1), S38-S81.

19. Bogdanov, S. (2002). Harmonized methods of the International Honey Commission Swiss Bee Research Centre, FAM, Liebefeld, CH 3003 Bern, Switzerland. 
20. Chirife, J., Zamora, M. C. \& Motto, A. (2006). The correlation between water activity and \% moisture in honey. Fundamental aspects and application to Argentine honeys Journal of Food Engineering, 72, 287-292.

21. Bogdanov, S., Martin, P., \& Lüllman, C. (1997). Harmonised methods of the European Honey Commission. Apidologie, extra issue, 1-59.

22. Rodriguez-Flores, M. S., Escuredo, O. \& Seijo, M. C. (2015). Assessment of physicochemical and antioxidant characteristics of Quercus pyrenaica honeydew honeys. Food Chemistry, 166, 101-106.

23. Martınez-Máñez, R., Soto, J., Garcia-Breijo, E., Gil-Sánchez, L., Ibáñez, J. \& Llobet, E. (2005). An “electronic tongue” design for the qualitative analysis of natural waters. Sensors and Actuators, B 104, 302-307.

24. Carpenter, G. A., Gossberg, S., Markuzon, N., Reynolds, I., \& Rosen, D. (1992). Fuzzy Artmap: a neural network architecture for incremental supervised learning of analog multidimensional maps. IEEE Trans Neural Network, 3, 698-713.

25. Llobet, E., Hines, E.L., Gardner, J.W., Bartlett, P.N. \& Mottram, T.T. (1999). Fuzzy ARTMAP based electronic nose data analysis. Sensors and actuators $b$ chemical, 61, 183-190.

26. Gil-Sánchez, L., Barat, J.M., Escriche, I., García Breijo, E., Martínez Mañez, R. \& Soto, J. (2008). An electronic tongue for fish freshness analysis using a thickfilm array of electrodes. Microchimica Acta. 163, 121-129.

27. Garcia-Breijo, E., Garrigues, J., \& Gil-Sánchez, L. (2013). Optimization of an Embedded Simplified Fuzzy ARTMAP Implemented on a Microcontroller using MATLAB GUI Environment”.Pattern Recognition: Practices, Perspectives and Challenges. Nova Science Publishers (Hauppauge NY, EEUU). 
González-Miret, M.L., Terrab, A., Hernanz, D., Fernández-Recamales, M.A. \& Heredia, F.J. (2005). Multivariate correlation between color and mineral composition of honeys and by their botanical origin. Journal of Agriculture and Food Chemistry, 6, 53(7), 2574-2580.

Karabagias, I. K., Vavoura, M.V., Nikolaou, C., Badeka, A. V., Kontakos, S. \& Kontominas, M.G. (2014). Floral authentication of Greek unifloral honeys based on the combination of phenolic compounds, physicochemical parameters and chemometrics. Food Research International, 62, 753-760.

Kasuba, T. (1993). Simplified Fuzzy ARTMAP. Al Expert, 18-25.

León-Ruiz, V., Vera, S., González-Porto, A. V. \& San Andrés, M. P. (2011). Vitamin C and sugar levels as simple markers for discriminating Spanish honey sources. Journal of Food Science, 76, C356-C361.

Bara MTF, Serrano SHP, Asquieri ER, Lúcio TC, Gil ES 2008. Medida del potencial anódico en estado sólido: Una herramienta para la determinación del potencial antioxidante de fitoterápicos. Lat Am J Pharm 27: 8992.

Blasco AJ, Gonzalez MC, Escarpa A 2004. Electrochemical approach for discriminating and measuring predominant flavonoids and phenolic acids using differential pulse voltammetry: towards an electrochemical index of natural antioxidants. Anal Chim Acta 511: 71-81.

Food and Nutrition Sciences, 2013, 4, 605-615 http://dx.doi.org/10.4236/fns.2013.46078 Published Online June 2013 (http://www.scirp.org/journal/fns)

The Joint Use of Electronic Nose and Electronic Tongue for the Evaluation of the Sensorial Properties of Green and Black Tea Infusions as Related to Their Chemical Composition Susanna Buratti1*, Antonella Casiraghi2, Paola Minghetti2, Gabriella Giovanelli1 1DeFENS, Department of Food, Environmental and Nutritional Sciences, Università degli Studi di Milano, Milano, Italy; 2Depart- ment of Pharmaceutical Sciences "Pietro Pratesi", Università degli Studi di Milano, Milano, Italy.

Email: *susanna.buratti@uni

\section{Figure captions}

Figure 1. PCA biplot (scores and loadings) of the variables (physicochemical parameters, sugars, color and total antioxidant activity) and honey samples: C (orange blossom); R 
(rosemary); T (thyme); S (sunflower); W (winter savory honey); H (honeydew honey). Next to each sample, the percentage of the relevant predominant pollen is shown.

Figure 2. PCA biplot (scores and loadings) from the measurements obtained with the 5 electrodes together (4 non-noble metals “Ag, $\mathrm{Ni}$, $\mathrm{Co}$ and $\mathrm{Cu}$ ”, plus $\mathrm{Au}$ ). $\mathrm{C}$ (orange blossom); R (rosemary); $\mathrm{T}$ (thyme); $\mathrm{S}$ (sunflower); $\mathrm{W}$ (winter savory honey); $\mathrm{H}$ (honeydew honey).

Figure S1. PCA biplot (scores and loadings) from the measurements obtained with the 8 electrodes together (4 noble and 4 non-noble metals: “Au, Pt, Ir, Rh, Ag, Ni, Co and Cu”). C (orange blossom); R (rosemary); T (thyme); S (sunflower); W (winter savory honey); H (honeydew honey).

Table 1. MLR prediction results for the physicochemical parameters (aw, conductivity, moisture), colour and antioxidant activity

\section{4 non-noble metals 4 non-noble metals}

+4 noble metals $\quad+$ Au

\begin{tabular}{|c|c|c|c|c|c|c|}
\hline Parameters & $\begin{array}{l}\text { Correlation } \\
\text { coefficient }\end{array}$ & Slope & Intercept & $\begin{array}{l}\text { Correlation } \\
\text { coefficient }\end{array}$ & Slope & Intercept \\
\hline aw & 0.4860 & 0.516 & 0.279 & 0.4790 & 0.445 & 0.319 \\
\hline Electrical conductivity & 0.9050 & 0.850 & 25.359 & 0.8959 & 0.813 & 51.126 \\
\hline Moisture & 0.7380 & 0.646 & 5.905 & 0.7040 & 0.558 & 7.311 \\
\hline Colour & 0.5300 & 0.634 & 14.529 & 0.7400 & 0.755 & 8.895 \\
\hline Antioxidant Activity & 0.9665 & 0.970 & 11.162 & 0.9660 & 0.911 & 22.256 \\
\hline
\end{tabular}


Table 2. MLR prediction results for the physicochemical parameters (aw, conductivity, moisture), colour and antioxidant activity

\begin{tabular}{|c|c|c|c|c|c|c|}
\hline \multirow[b]{2}{*}{ Parameters } & \multicolumn{2}{|c|}{$\begin{array}{l}4 \text { non-noble metals } \\
\text { +4 noble metals }\end{array}$} & \multicolumn{3}{|c|}{$\begin{array}{l}4 \text { non-noble metals } \\
+\mathrm{Au}\end{array}$} & \multirow[b]{2}{*}{ Intercept } \\
\hline & $\begin{array}{l}\text { Correlation } \\
\text { coefficient }\end{array}$ & Slope & Intercept & $\begin{array}{l}\text { Correlation } \\
\text { coefficient }\end{array}$ & Slope & \\
\hline aw & 0.4860 & 0.516 & 0.279 & 0.4790 & 0.445 & 0.319 \\
\hline Electrical conductivity & 0.9050 & 0.850 & 25.359 & 0.8959 & 0.813 & 51.126 \\
\hline Moisture & 0.7380 & 0.646 & 5.905 & 0.7040 & 0.558 & 7.311 \\
\hline Colour & 0.5300 & 0.634 & 14.529 & 0.7400 & 0.755 & 8.895 \\
\hline Antioxidant Activity & 0.9665 & 0.970 & 11.162 & 0.9660 & 0.911 & 22.256 \\
\hline
\end{tabular}

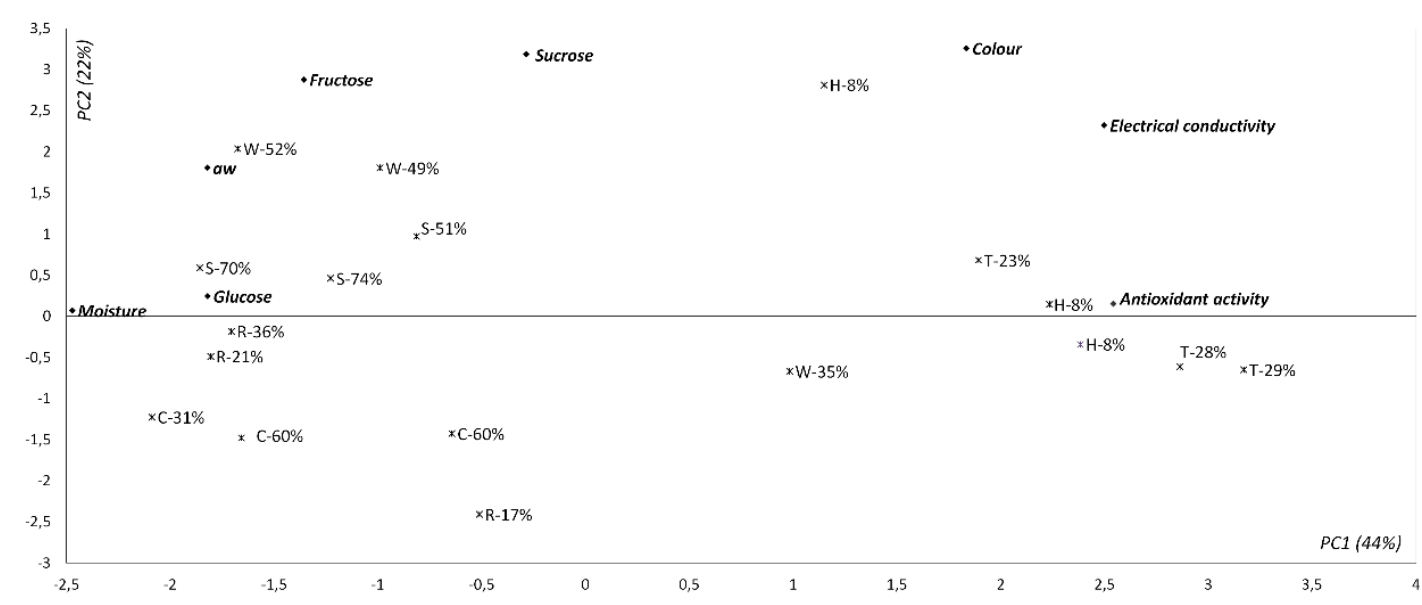

Figure 1 


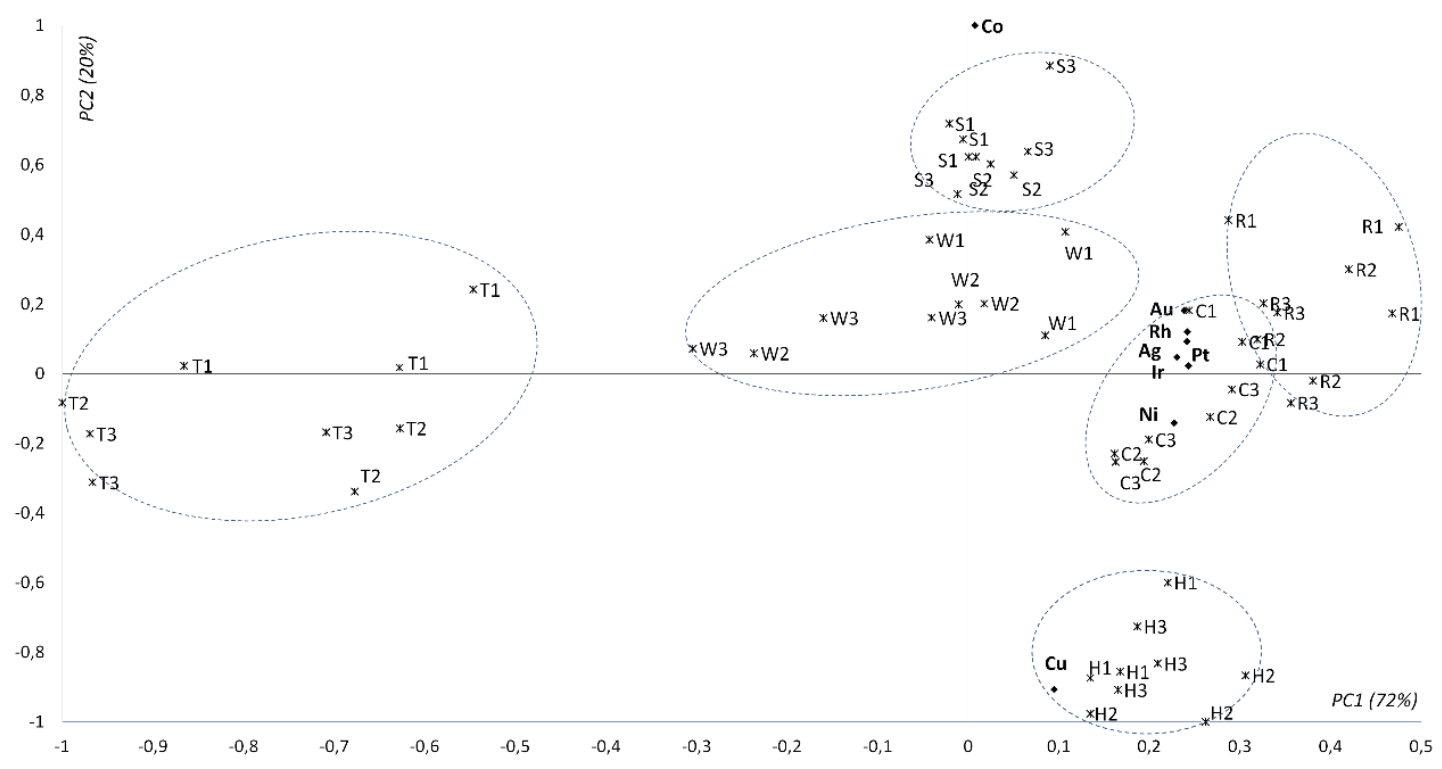

Figure 2

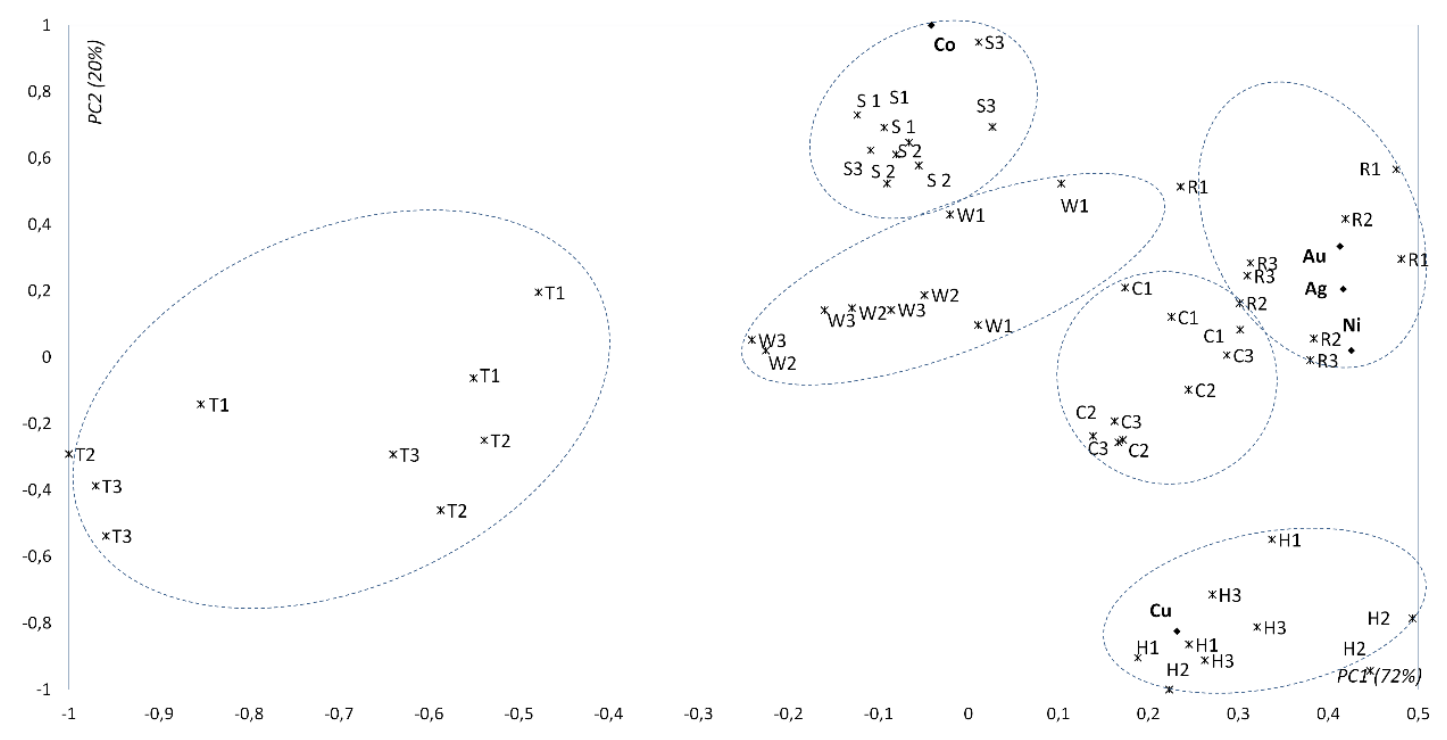

Figure 3 